\title{
Rechtsgeschichte
}

http://www.rg-rechtsgeschichte.de/rg2

$\operatorname{Rg} 22003 \quad 72-86$

Zitiervorschlag: Rechtsgeschichte Rg 2 (2003)

http://dx.doi.org/10.12946/rg02/072-086

\section{Luca Loschiavo}

\section{La legge che dio trasmise a Mosè}

Fortuna medioevale di un'operetta volgare 


\section{Abstract}

Even today the authorship and the date of the Collatio legum Mosaicarum et Romanarum or Lex Dei continues to be debated. It has often been maintained that the author intended to convince a pagan readership of both the authoritative character and the historical priority of theMosaiclaw over thelaw of Rome. An older thesis, however, maintained that the function of the work was to make available a text which would be useful to bishops of the ancient Christian communities in their function as judges. A similar need is effectively documented in the post-Constantinian Church. The writing of the Collatio, in substance, could represent an attempt to integrate Roman legal culture into Christianity.

Whatever may have been the origin of this vulgar legal work, no less interesting - albeit less researched - is the problem of the fortune of the Lex Dei in the Middle Ages. One may ask precisely for what reasons and according to what means a text of this sort could have a different fortune from that experienced, for example, by the Fragmenta Vaticana, and yet could still return to circulate after more than four centuries of apparent oblivion.

The manuscript tradition of the Collatio is entirely medieval. The three manuscripts which have survived are all datable between the ninth and eleventh century. In addition, medieval texts are not lacking which offer significant confirmation of the knowledge of this text throughout that period. More or less literal citations of the LD emerge in canonistic texts from the ninth century onwards. One can even advance the hypothesis that, before the Bolognese "renaissance» elevated the Justinianic texts above all other Roman legal writings, the mysterious Pepo may have had knowledge of the Collatio. 


\section{La legge che dio trasmise a Mosè}

Fortuna medioevale di un'operetta volgare

I È stato Ludwig Schmugge (nr. 54), tredici anni fa, a individuarlo e renderlo noto agli studiosi. L'intera questione relativa all'affascinante personaggio di Pepo è ora ampiamente e criticamente riesaminata da E. CORTESE (nr. 8: I, 383 e sgg.; II, 33-55). Recentissimamente è tornata $\mathrm{a}$ scriverne anche G. NicOlaJ (nr. 46: in partic. 49I-2).

2 Riporto, per comodità del lettore, la parte saliente del breve passaggio già edito dallo ScHMUGGE (nr. 54:3) con la piccola variazione suggerita dallo Spagnesi e accettata dal CORTESE (nr. 8: II, 3678): "... Surrexit autem magister Peppo in medium tantum Codicis Iustiniani et institutionum bàiulus, utpote Pandecte nullam habens noticiam,... enervans sentenciam priorum iudicum. Quippe allegavit eum, qui exemisset hominem de grege hominum, universitati fore iniurium adeo, ut qui hominem ademisset universitati hominum, quia violasset naturale communionis consorcium, ipse pariter de medio tolleretur et homicida occideretur. Sive enim servus sive liber foret, idem ait esse iudicium, quoniam addictio servitutis delere non poterat communionem nature humane conditionis. Legibus igitur et sacris constitutionibus imperatorum firmato iudicio optinuit magister Peppo coram imperatore aliis iudicibus in confusione recedentibus«.

3 Cfr. l'ulteriore passo riportato dallo SCHMUGGe (nr. 54) 4.

4 Ai passi già segnalati dal DolcinI (nr. I2: in partic. 87-90 e ivi le ntt. I3, I4, I8), potrebbe anche aggiungersi quel brano della Pro Milone (6.I7) in cui Cicerone sottolinea l'indifferenza della condizione sociale della vittima nella valutazione dei casi di omicidio: "Quid ita? Quia non alio facinore clari homines, alio obscuri necantur. Intersit inter vitae dignitatem

mors quidem inlata per scelus is-

I. Cominciamo dalla fine. Il passo in cui Radulfus Niger racconta dell'intervento del misterioso Pepo o Pepone nel placito tenutosi in Lombardia alla presenza di Enrico IV è divenuto ormai famoso. ${ }^{\mathbf{I}}$ Quanti si sforzano di gettare luce sulle origini del rinascimento giuridico medievale, lo hanno letto e riletto indagandone anche i minimi dettagli. Eppure, ogni ulteriore lettura di quel passo sembra proporre motivi di interesse e spunti di riflessione sempre nuovi.

La vicenda è notissima. ${ }^{2}$ Radulfus ci presenta il magister Peppo nell'atto di reagire energicamente alla comminazione di una pena pecuniara nei confronti dell'uccisore di un servo. A giudizio dell'antico maestro, sebbene espressamente prevista per casi del genere dalle vigenti leggi longobarde, una semplice multa non può in ogni caso esser sufficiente a sanzionare un delitto come l'omicidio: i giudici avrebbero senz'altro dovuto comminare la pena capitale. L'episodio - è lo stesso Radulfus a chiarirlo poco oltre - dovrebbe esemplificare l'atteggiamento della Chiesa riformatrice ormai apertamente contraria al giudizio di tipo germanico, imperniato sulle compositiones e su sistemi probatorî ordalici. ${ }^{3}$ Piuttosto si potrebbe rimanere sconcertati nel vedere l'imperatore così pronto a recepire un discorso dai toni in verità un po' inquietanti e, da un punto di vista di stretta legalità, alquanto eversivo.

Sono le argomentazioni prodotte da Pepo a sollevare, in effetti, i maggiori problemi. Due, in particolare, i principî sui quali egli costruisce il proprio ragionamento. Per il primo, il diritto naturale richiede che l'uccisione ingiustificata di un uomo sia senz'altro punita con la morte dell'uccisore. In secondo luogo, la modifica dello status personale (cioè l'addictio servitutis) non incide sulla naturale condizione umana e quindi non giustifica deroga alcuna al principio precedente. Si è creduto di poter avvertire nel discorso di Pepo lontani echi ciceroniani. ${ }^{4} \mathrm{Si}$ è anche pensato a letture patristiche. ${ }^{5}$ Ennio Cortese ha tuttavia fatto notare come l'aurora surgens della rinata iuris disciplina - come pomposamente il Niger definisce Pepo $^{6}$ - sembri comunque richiamarsi più alla biblica >legge del taglione che non al maturo e raffinato diritto penale romano. ${ }^{7}$ Soprattutto, e ciò del resto ben si accorda con entrambe le altre testimonianze che del magistero di Pepo sono giunte sino a

dem et poenis teneatur et legibus. Nisi forte magis erit parricida, si qui consularem patrem quam si quis humilem necaverit ..."

5 Dolcini (nr. I2) 89-90 e, in particolare, 87I5-I 6 .

6 In un altro passo dei Moralia Regum che aveva, a suo tempo, già individuato il KANTOROWICZ (nr. 25).

7 Cortese (nr. 8: II, 39; nr. 9: IO5O-I). Pepo avrebbe potuto tener presente anche Exod. 2 I.20 (magari nella versione un po' differente di cui si dirà infra nt. I9): »Qui percusserit servum suum vel ancillam virga, et mortui fuerint in manibus eius, criminis reus erit «. Si vedano, d'altro canto, i passi del Digesto ricordáti più avanti (infra nt. I6) in cui è ripetuto il principio liberum corpus aestimationem non recipit. 
noi, ${ }^{8}$ è Isidoro di Siviglia la sua fonte principale, quella da cui trae l'idea della appartenenza della legge del taglione al diritto di natura. ${ }^{9}$ Non si può certo dire che l'ispirazione che muove Pepo sia quella del romanista rigoroso.

Tuttavia il Niger - e non vi è motivo per dubitare qui della nostra fonte - ci dice pure che il vecchio bàiulus del Codice e delle Istituzioni avrebbe firmato il suo discorso allegando leges e sacrae constitutiones imperatorum. È alle leggi romane, evidentemente, che Radulfus intende riferirsi. Sappiamo, in effetti, come, sullo scorcio del secolo XI, il diritto romano andasse aprendo crepe sempre più ampie nel sistema normativo longobardo-franco. Papi e imperatori venivano progressivamente accorgendosi di quale formidabile strumento di potere esso potesse divenire in mani appositamente addestrate. ${ }^{\text {Io }}$ E pertanto del tutto naturale che Pepo cercasse di far breccia nel cuore di Enrico IV attraverso altisonanti citazioni di antiche leggi e augusti predecessori. Se fu infatti Isidoro a fornirgli l'ispirazione principale, è chiaro che ben difficilmente Pepo sarebbe riuscito a scardinare agli occhi dell'imperatore un giudizio tecnicamente ben confezionato (multiplici allegatione iuris) dai giudici regi, appoggiandosi sulla sola autorità di quel manuale enciclopedico che erano le Etymologiae del vescovo spagnolo. Quel suo ragionamento, così >rivoluzionario<, avrebbe potuto aver successo solo contrapponendo alle norme longobarde auctoritates giuridiche indiscutibilmente superiori: la legge divina innanzi tutto e, appunto, il diritto romano cui nessuno - nemmeno fra i longobardisti - pensava di poter contestare la qualifica di lex omnium generalis.

Ma quali potrebbero essere, in concreto, le norme romane richiamate in quel placito? Quelli sono appunto gli anni in cui, nelle medesime contrade dell'Italia centro-settentrionale che vedono attivo Pepo, ricordi e citazioni dei volumina giustinianei si facevano sempre più numerosi e puntuali. Benché non numerosissimi, la compilazione contiene nondimeno alcuni passi che il misterioso Pepo avrebbe potuto utilimente allegare a sostegno del suo ragionamento.

Egli conosceva certamente le Istituzioni e il Codice sui quali, chissà dove, teneva lezioni. Attraverso le prime Pepo avrebbe potuto citare la lex Cornelia de sicariis »quae homicidas ultore ferro persequitur « (Inst. 4.I 8.5). ${ }^{\text {II }}$ Sempre un passo delle Istituzioni ricordava poi come la lex Aquilia de damno consentisse al

8 Cioè i due tentativi etimologici relativi il primo al termine $m u$ tuum e contenuto nella antica Summa Institutionum provenzale edita dal LEGENDRE (nr. 3 I) (cfr. in proposito CORTESE; nr. 9: I049-50), il secondo all'espressione ante felicem embolam di C. I.2.Io.pr. (su cui Loschiavo; nr. 36: 233 e sgg.).

9 Come ha osservato il Cortese (nr. 8: II, 37-38 e 40), indicando soprattutto Isidorus, Etymol., $5 \cdot 4$. I e $5 \cdot 27 \cdot 24$. In quest'ultimo passo, in particolare, Isidoro accosta il talio alla vindicta ("... talio est similitudo vindictae $«)$ per poi affermarne la rispondenza alla legge di natura e a quella divina (mosaica) (»... hoc enim est natura et lege institutum, ut laedentem similis vindicta sequatur «). Almeno in parte, Isidoro potrebbe a sua volta aver tratto ispirazione da Cicerone che in $\mathrm{De}$ invent. 2.65 aveva appunto collocato la vindicatio all'interno del diritto naturale: "... ac naturae quidem ius esse, quod nobis non opinio, sed quaedam innata vis afferat, ut religionem pietatem gratiam vindicationem observantiam veritatem ... vindicationem, per quam vim et contumeliam defendendo aut ulciscendo propulsamus ... «; sui significati di questo passaggio ciceroniano, cfr. KUNKEL (nr. 28) I 26 e sg.

Io Val la pena di riportare anche qui le parole di Radulfus Niger (il passo è quello già ricordato supra, nt. 6) secondo il quale, proprio nell'epoca che fu di Pepo e poi di Irnerio, il diritto civile (cioè il romano) "traheretur ad curiam romanam, et in aliquibus partibus terrarum expanderetur in multa veneratione et munditia, ceperunt leges esse in honore simul et desiderio, adeo ut occideretur Amon, abrogato pravo ritu iudiciorum in plerisque partibus terrarum. Sed et quamquam ab initio displicerent iura principibus, quia vetustas consuetudines erasissent, tandem tamen ecclesia procurante et propagante eorum scientiam, usque ad principes produxerunt eorum notitiam, et apud eos invenit eis gratiam «.

I I Come indicato dal Cortese (nr. 8: II, 39). Su questo testo legislativo di epoca sillana si veda in partic. FERRARY (nr. I4). Più in generale, sul ius talionis nel mondo antico, cfr. HerdlitczKa (nr. 23). 
I 2 "Liberum est autem ei, cuius servus fuerit occisus, et privato iudicio legis Aquiliae damnum persequi et capitalis criminis eum reum facere «; passo individuato dal Dolcini (nr. I2: 88I9).

I3 La segnalazione si deve ancóra al Cortese (nr. 8: II, 37).

I4 Che si sostanziava nella deportatio per gli honestiores e nella morte per gli humiliores, cfr. PS 5.23.I. Sulla repressione dell'omicidio con la pena capitale in diritto romano, si vedano Mommsen (nr. 4I: 6 I 2 e sgg.), LeVy (nr. 33: 325 e sgg., partic. 357) e quindi Santalucia (nr. 53: I45-9 e 26I-3). Sulla distinzione humiliores/honestiores e sui risvolti che essa aveva anche a questo proposito, cfr. RILINGER (nr. 5I: partic. I 8I-206).

I 5 Proprio le Istituzioni di Giustiniano spiegano, in realtà, come il ius talionis, già conosciuto e applicato all'epoca delle XII Tavole, sarebbe presto divenuto desueto (Inst. 4.4.7). Anzi - si veda nuovamente Cortese (nr. 8: II, 39) -, una costituzione di Diocleziano (C. 2.4.I 8) - che però si sospetta interpolata - invitava a non comminare con troppa facilità la punizione estrema al di fuori dei casi di adulterio.

I6 Niente, infatti, sembra smentire la precisa affermazione del Niger secondo cui Pepo non avrebbe avuto conoscenza diretta del Digesto (... Pandecte nullam habens noticiam ...). Analogamente, anche se in questo secondo caso si sarebbe trattato del Digestum vetus ormai prossimo alla $>$ riscoperta $<$ Pepo non avrebbe potuto citare nemmeno D. 9.2.23.9: "Si dolo servus occiso sit, et lege Cornelia agere dominum posse constat: et si lege Aquilia egerit, praeiudicium fieri Corneliae non debet «; cfr. DolcINI (nr. I2: 88I8-I9). Va peraltro sottolineato come il Digestum vetus contenga fra l'altro ripetute menzioni del principio liberum corpus aestimationem non recipit (D. 9.1.3; D. 9.3.7; D. I 4.2.2.2) che invece, ammettendo implicitamente l'aestimatio corporis per i servi, sarebbero state certamente di ostacolo alla costruzione peponiana. Sempre il Digesto accenna secondo il diritto naturale prima

padrone del servo ingiustamente ucciso di agire per il risarcimento del danno e al tempo stesso di intentare contro l'uccisore un giudizio per omicidio (Inst. 4.3.II). ${ }^{\mathrm{I} 2}$ Dal Codice avrebbe quindi potuto trarre un breve passaggio forse ancor più rispondente ai suoi bisogni. In una costituzione del 385 , sia pure in maniera vaga, Graziano e Teodosio sembrano infatti alludere al ius talionis proprio nella repressione dell'omicidio (C. I.4.3.3): » ... homicida et parricida quod fecit semper expectet $«{ }^{\mathbf{I} 3}$

Allegando questi passi, in sostanza, Pepo avrebbe potuto dimostrare principalmente due cose. La prima è che, secondo il diritto romano, il padrone del servo ucciso era abilitato a sollevare una autentica accusa criminale nei confronti dell'omicida. La seconda è che le leges romane prevedevano che l'omicidio fosse senz'altro punito con la pena capitale ${ }^{\mathrm{I} 4} \mathrm{e}$ che - forzando un po' il significato delle norme - nemmeno l'applicazione della legge del taglione era del tutto sconosciuta. ${ }^{15}$ Non è esattamente la piena conferma del suo argomentare ma, rivolgendosi a un pubblico educato a una logica giuridica non proprio raffinata e - si può immaginare - pressoché digiuno di diritto romano, a Pepo poteva forse essere sufficiente.

Eppure il Corpus iuris contiene ancóra una norma che si sarebbe adattata ottimamente al caso e avrebbe completato mirabilmente il quadro di puntelli normativi su cui poggiare la logica un po' esaltata di Pepo. Si tratta di un frammento del giurista Marciano, il quale, commentando la ricordata lex Cornelia, fra l'altro scrive: »Et qui hominem occiderit, punitur non habita differentia, cuius condicionis hominem interemit «. Il passo appartiene tuttavia ai libri terribiles del Digesto (D. 48.8.I.2) e quindi l'ipotesi che Pepo abbia potuto conoscerlo e citarlo parrebbe senz'altro da escludere. ${ }^{\text {I6 }}$

È anche possibile, tuttavia, che Pepo potesse disporre di fonti giuridiche romanistiche estranee alla compilazione di Giustiziano. Un sospetto - in verità assai fugace - potrebbe già prendere corpo di fronte a un passo delle Pauli Sententiae ripreso nel Breviarium alariciano e di lì passato nei Libri de synodalibus causis di Reginone di Prüm: in esso, sia pure riferite ad altra fattispecie criminosa, ritroviamo infatti quasi le identiche parole (... sive is servus sive liber sit, capite punietur ...) che il Niger mette in bocca a Pepo. ${ }^{{ }^{17}}$ Ma che Pepo potesse conoscere il Breviarium o l'opera di Reginone è, in realtà, un eventualità anch'essa piuttosto improbabile. ${ }^{\text {I8 }}$

che il ius gentium fosse intervenuto a modificarla.

I7 PS 5.23.I 3 che nel Breviarium diviene 5.25 .7 (nr. 20: 436) e in Reginone corrisponde al cap. 87 del secondo libro (nr. 64: 247). Al Dolcini (nr. I 2: 87I 7 ) è parso piuttosto che la derivazione potesse essere dalla lettera di Paolo agli Efesini (Ad Eph. 6.8).

I $8 \mathrm{Si}$ ricordi peraltro come anche il Brachylogus iuris civilis - un'ope- ra sulla quale, al di là dell'antica attribuzione del Naber, si proietta stranamente anche l'ombra di Pepo - rivela pur'esso citazioni tratte dalla Lex Romana Wisigothorum (cfr. CoRTESE; nr. 8: II, 52-55). Quanto all'opera di Reginone, dopo aver ricordato come lo scarso numero di manoscritti conservatisi lascia credere che essa abbia avuto un successo piuttosto limitato, occorre sottolineare come il 
Il sospetto assume invece contorni ben più concreti e definiti non appena si prende a leggere quell'operetta tardoantica che ci è nota come Lex Dei o Collatio legum Mosaicarum et Romanarum. L'originale tentativo di comparazione tra le norme mosaiche e il diritto romano comincia, infatti, proprio dal reato di omicidio. Già nell'impostazione la Collatio risultra perfettamente congeniale al discorso condotto da Pepo innanzi all'imperatore. Al principio veterotestamentario (lex Dei) secondo cui chi cagiona la morte deve essere a sua volta ucciso (qui, in particolare, si cita Num. 35.I6-I7, 20-2I), l'ignoto autore contrappone una pluralità di fonti giuridiche romanistiche, appunto nel tentativo di metterne in risalto la corrispondenza. In particolare, egli riporta qui alcuni passi relativi alla lex Cornelia estratti dagli scritti di Paolo e Ulpiano (Coll. I.2-4). È soprattutto il frammento ulpianeo ad attirare l'attenzione dal momento che, a ben guardare, esso avrebbe consentito a Pepo di ovviare alla sua ignoranza del Digesto. Ulpiano, infatti, ripropone lo stesso principio dell'antica legge romana riferito da Marciano nel frammento di cui si è detto prima e che sarebbe tornato così utile nel placito lombardo (Coll. . $.3 .2=$ Ulp. lib. VII de officio proconsuli sub tit.de sicariis et veneficis):

... Conpescit item eum, qui hominem occidit, nec adiecit cuius condicionis hominem, ut et ad servum et peregrinum pertinere haec lex videatur.

Potendo disporre di un testo come la Collatio - che oltretutto contiene anche un titolo (il terzo) specificamente dedicato ai maltrattamenti arrecati ai servi ${ }^{19}$ - Pepo avrebbe quindi avuto modo di completare la serie di auctoritates romanistiche con le quali firmare il suo iudicio e lasciare in confusione gli altri giudici al cospetto dell'imperatore. Inoltre, cosa ancor più importante, avrebbe potuto dimostrare che quanto egli andava affermando non era affatto il frutto di una sua personale elucubrazione. Né si trattava di una costruzione edificata unicamente sulla base di autorità letterarie e patristiche. Era, al contrario, un'autentica raccolta giuridica a ssancire la perfetta consonanza esistente a quel proposito fra la legge che Dio stesso aveva dettato a Mosè (e che, nella visione cristiana, era al tempo stesso diritto divino e naturale) e il diritto universale per eccellenza, ${ }^{20}$ quel diritto di Roma, cioè, della cui applicabilità in un giudizio tenuto dall'autorità imperiale non doveva dubitarsi.

passo delle Sententiae in questione non sia stato recepito né da Burcardo né da Ivo di Chartres ai qualli si deve, per converso, la grande influenza che essa ebbe ne secolo XI; cfr. in proposito FouRNIER, LE BRAs (nr. I7) 267 e sg.

I9 È interessante notare come la versione di Exod. 2 I.20 proposta da Coll. 3.I (»Si quis percusserit servum aut ancillam uirga et mortuus fuerit in manibus eius, iudicio vindicetur ... «) differisca da quella della Vulgata (v. supra nt. 7) e come, in particolare, manchi qui la specificazione suum che avrebbe potuto ostacolarne l'impiego da parte di Pepo in occasione del placito lombardo. Cfr. inoltre, quale corrispondente romanistico, Coll. 3.2.I: "Servus si plagis defecerit, nisi id dolo fiat, dominus homicidii reus non potest postulari ...«(= Paulus 1. sent. V sub tit. ad legem Corneliam de sicariis et veneficis).

20 Forse non è un caso che Pepo, nel discorso riferitoci da Radulfus, ponga due volte l'accento sul danno arrecato all'universitas degli uomini. Al Dolcini (nr. I 2: 90) tale richiamo è parso il contributo più originale offerto in questo frangente dall'antico giurista, l'unico che " potrebbe risalire all'autonoma elaborazione di pensiero da parte di Pepo«. 
2I Non è lecito invece nutrire dubbi riguardo alla circostanza che nella società altomedievale la legge mosaica continuasse ad avere grande importanza e le fosse attribuita immediata efficacia normativa. Non è da escludere, per fare un esempio, che proprio alla legge mosaica si facesse riferimento con la generica espressione lex nostra talvolta riscontrabile anche nelle fonti giuridiche; cfr. a tale proposito SIEMs (nr. 58: 327 nt. 808). Ma questo, appunto, è un altro discorso.

22 Nessuno, oggi, si preoccupa più di legare quest'operetta a un nome specifico. Una rapida rassegna dei varî tentativi fatti in passato in SCHRAGe (nr. 55:329-33I).

23 Senz'altro importanti in questo senso sono però da considerare $\mathrm{i}$ risultati cui è giunto lo SCHRAGE

Ci si può chiedere, a questo punto, se sia effettivamente ipotizzabile una conoscenza della Lex Dei da parte di Pepo. ${ }^{2 \mathrm{I}} \mathrm{Si}$ vedrà che una simile ipotesi è assai più verosimile di quanto non possa sembrare a prima vista. È il momento di abbandonare il placito lombardo e rivolgersi alla storia di questa operetta così peculiare.

2. Della Lex Dei quam praecepit Dominus ad Moysen - questo è propriamente il titolo che si ricava dai manoscritti - sappiamo in realtà assai poco. Non ne conosciamo l'autore, ${ }^{22}$ non l'esatta datazione $^{23}$ e nemmeno, al di là delle diverse ipotesi formulate, possiamo esser veramente sicuri della sua redazione in ambiente ebraico o, altrimenti, cristiano. ${ }^{24} \mathrm{Nel}$ porre di fronte la legge mosaica e il diritto romano in un sistematico confronto, questa operetta costituisce certamente uno fra i primissimi esempî di comparazione tra due sistemi giuridici. Soprattutto, però, essa è anche una delle poche fonti della giurisprudenza romana pregiustinianea che siano giunte sino a noi.

Non può sorprendere quindi che, dal momento della sua riscoperta e della sua prima edizione avvenuta nel I 574 ad opera dell'umanista francese Pierre Pithou, ${ }^{25}$ la Collatio sia divenuto un tradizionale campo di ricerca per gli storici del diritto romano. Assai meno comprensibile riesce invece il silenzio che, salvo poche eccezioni, hanno tributato a questa operetta gli studiosi del diritto medievale. Eppure, a pensarci bene, la tradizione manoscritta della Collatio è tutta medievale e, sempre all'alto Medioevo, appartengono anche le poche tracce che son parse sino ad ora documentare un qualche impiego di quel testo. Sarebbe stato quindi del tutto naturale investigare le vie attraverso le quali quell'operetta volgare fu tratta nuovamente alla luce dopo circa quattro secoli di oblío. Soprattutto, però, incuriosiscono i motivi che indussero a fare delle nuove copie di quell'insolito testo, iniziandone così una circolazione che fu tutt'altro che modesta e che, per quanto ne sappiamo, si estese dal secolo IX sino allo scorcio dell'XI interessando l'Italia e la Francia.

La Lex Dei - benché si sia cercato di negarlo ${ }^{26}$ - era certamente conosciuta da Incmaro arcivescovo di Reims. Lo rivelano due passaggi del De divortio Lotharii regis et Thetbergae reginae che egli scrisse intorno all'anno 860. In una prima circostanza Incmaro ricorda un passo del Levitico - lo stesso con cui si apre il tit. de stupratoribus della Collatio (Coll. 5.I) - e poi ne segnala la

zione dell'operetta: l'autore della Collatio sarebbe appartenuto all'epoca e all'ambiente che furono di S. Agostino.

$24 \mathrm{Fu}$ il Volterra, nel I930, in un lavoro che impressiona per la vastissima erudizione (nr. 63), a riprendere con molti nuovi argomenti una tesi, quella della matrice ebraica della Lex Dei, che era già stata avanzata più volte a partire dal XVI sec. Tale tesi fu successi- vamente accolta dal Gaudemet e riproposta dagli allievi del VoLTERRA (puntuali indicazioni in Liebs; nr. 34: I63 e sg.). Da ultimo l'ha ripoposta BARONE-ADESI (nr. I).

25 La scoperta del manoscritto su cui l'umanista francese basò la sua edizione avvenne anteriormente al I 572 (Volterra; nr. 63: 8 e sg.).

26 Cfr. in particolare VolterRA (nr. 63) 23-3 I. 
convergenza con le norme romane che il vescovo trovava raccolte in un apposito capitolo, appunto intitolato de stupratoribus. ${ }^{27}$ Più oltre Incmaro rinvia nuovamente a quanto disposto dai capitoli de stupratoribus e de incestis et turpibus nuptiis che egli dice essere il sesto e il settimo del primo libro di una non meglio precisata Lex Romana. ${ }^{28}$ Tra le fonti romanistiche a noi note, titoli di ugual tenore e in tale successione si trovano solamente nella Lex Dei. ${ }^{29}$ L'incidentale quod legens quisque inveniet che Incmaro fa seguire al primo rinvio, lascia poi intendere come il vescovo non ritenesse affatto difficile la reperibilità di questi testi e quindi, presumibilmente, della stessa Collatio: è un'indicazione da non trascurare soprattutto perché sembra essere supportata da ulteriori indizî.

Al Mommsen, per cominciare, parve di avere individuata una interessante conferma in un manoscritto della Lex Romana Visigothorum conservato a Parigi (Paris, BN lat. 9652) e risalente anch'esso, al pari dello scritto di Incmaro, al sec. IX. Nell'ultima carta di quel codice, una mano di poco posteriore a quella che ha copiato il Breviarium ha infatti trascritto il versetto biblico di Ex. 22.7 premettendovi l'iscrizione »X. De deposito «: esattamente in questo modo inizia appunto il decimo titolo della Collatio. ${ }^{30}$

Più tardi, cioè nei primi decenni del sec. XI, anche l'autore della >Collezione canonica in cinque libri ${ }^{3 \mathrm{I}}$ sembra utilizzare la Lex Dei. In particolare, inframezzato a una corposa serie di estratti dall'Epitome Iuliani, egli riproduce un passo delle Sententiae di Paolo (PS 5.23.3) che non è fra quelli presenti nel Breviarium Alaricianum e che è invece contenuto nella Collatio (7.I). ${ }^{32}$ Il fatto che nella rubrica il passo sia attribuito a Iustinianus rex si spiega facilmente constatando come la medesima inscriptio sia costantemente preposta anche ai molti excerpta dall'Epitome Iuliani fra i quali, come s'è detto, si trova inserito. ${ }^{33}$ È noto, del resto, come in Italia - patria certa della collezione canonica - usasse riferire a Giustiniano ogni testo giuridico romanistico all'epoca in circolazione. ${ }^{34}$ La Collectio V librorum, oltre a citare questi testi di diritto romano e altre norme longobardo-franche, si segnala anche per la massiccia presenza di citazioni dalla Bibbia e in particolare dal vecchio Testamento: una circostanza piuttosto inconsueta che ha fatto pensare all'influenza delle raccolte canoniche di origine irlandese e, in particolare, della Hibernensis. ${ }^{35}$

Tre sole attestazioni - pur niente affatto fragili e insignificantinon sono certo molte e non potrebbero consentire alcuna sicurezza

27 Interrog. I 2, resp. (ed. Sirmond, PL I 25, 690 sub B): »Et scriptum est in libro Levitici: Qui dormierit cum masculo coitu femineo, uterque operatus est nefas, morte moriantur (Lev. 20.13). Unde et leges Romanae decernunt in capitulis de stupratoribus, quod legens quisque inveniet ".

28 Ivi, 697 sub B: "... sicut in primo libro legis Romanae capitulo sexto de stupratoribus, et in capitulo septimo de incestis et turpibus nuptiis praecipitur, et in caeteris, quae christiana iura depromunt, iusti iudices legere possunt «.

29 La tradizione manoscritta - come già il Mommsen (nr. 42: I I 2 ) aveva notato - consentirebbe inoltre di spiegare sia l'indicazione liber primus (che è infatti presente nel ms. viennese all'inizio della Collatio) sia lo sfasamento nella suddivisione dei titoli (i titt. citati da Incmaro per noi sono in realtà il quinto e il sesto; nel manoscritto berlinese, prima di Coll. 5.I, si legge invece [fol. I65r]: »Explicit titulo quinto. Incipit de stupratoribus«). È pure possibile che, nei secoli altomedievali, circolasse anche qualche altra versione della Lex Dei un po' differente da quella a noi pervenuta. Cfr. inoltre CoNRAT (nr. 6: 88I) e ora LiEBS (nr. 34: I623).

30 Mommsen (nr. 42: I I4) su cui, criticamente ma senza argomenti veramente decisivi, VolterRA (nr. 63: 35-6). Sul manoscritto si veda Dolezalek (nr. I3: II, adv.).

3 I La collezione fu composta quasi certamente a Roma (o nell'Italia meridionale) tra il IOI4 e il IO23 (con maggior probabilità intorno al ı020) all'epoca, cioè, della stretta collaborazione tra Enrico II e il papa Benedetto VIII; cfr. FoURNIER (nr. I6: 277-307 e, partic., 300-3 sulle fonti normative secolari in essa utilizzate, 304-7 sull'origine e la datazione) e Supino Martini (nr. 6I: 205 I4 sull'origine del cod. Vallic. B I I e 226-9 sul ms. Vat. lat. I339). Più recentemente, è giunto sostanzialmente alle medesime conclusioni anche Koal (nr. 27: I27-I34). Cfr. inoltre KÉRY (nr. 26: I 57-I60).

32 Il passo è anche in questo caso relativo all'omicidio e, nella Collectio canonum, si trova inserito sotto la rubrica »de homicidiis qui aliquando absolvuntur, aliquando damnantur. Iustinianus rex «. Cfr. Mommsen (nr. 42: I I3-4) e Conrat (nr. 6: 88 I e 2I 5-6).

33 Contra Volterra (nr. 63: 33).

34 Che l'attribuzione a Giustiniano di testi giuridici non suoi sia un valido argomento per riconoscere la provenienza italiana di opere altomedievali è stato sostenuto dal Patetta (nr. 47: 870-I) e, più di recente, dal CORTESE (nr. 8: I, 834 e 253).

35 Cfr. Patetta (nr. 48: i7-2I) e FOURNIER (nr. I6). Si veda inoltre Mor (nr. 43: in partic. I77-9). 
36 V. in partic. SChultz (nr. 56) ripreso ora anche da NELSON, MANTHE (nr. 44: 9).

37 Cfr. RöHLE (nr. 52: I36I35), ove è anche riportata l'opinione del Bischoff secondo cui il fascicolo sarebbe appunto da attribuire alla prima metà del sec. IX, alla Francia sud-orientale o all'Italia settentrionale.

38 Il manoscritto è stato descritto da HÄNEL (nr. 2I: iv-vi) e ora nuovamente da NeLson e MANTHE (nr. 44: I 5-I 8) che pensano a un'origine nella regione retica $o$ nell'Italia settentrionale o anche nella Francia del Nord. Se quest'ultima sembra però senz'altro da escludere (v. RöHLE; nr. 52 : I 29), Nicolaj (nr. 46: I36) pensa in particolare al territorio nonantolano.

39 Descrizioni del codice in Bluhme (nr. 2: 3 I I) e soprattutto HäNEL (nr. 2I: vi-vii); cfr. inoltre DoleZAleK (nr. I3) $a d v$. e Nelson, Manthe (nr. 44: 20-22 con datazione alla prima metà del sec. IX). Su di esso è tornato da ultimo Ciaralli (nr. 4: ntt. 7-I4) che, fondandosi sugli studi condotti della Supino MARTini intorno alla scarolina romana (nr. 6I) vi ha appunto riconosciuto un esempio di quella particolare scrittura.

40 Cfr. Bluhme (nr. 2: 3 I I ); Hänel (nr. 2I: vii-viii); Dolezalek (nr. I3) ad v.; Nelson, Manthe (nr. 44: 19-20).

4I Già il Patetta (nr. 49: I26-I3I), sottolineando i significativi legami con la Summa Perusina, riteneva probabile una stesura del manoscritto in ámbito romano. Ciaralli (nr. 4 : ntt. 35 e sgg.) riconosce ora anche in questo codice un ulteriore esempio di sminuscola romanescar e, con buoni argomenti, propone di ritardare leggermente la sua redazione collocandola nella seconda metà del secolo XI. Datazione completamente differente (metà del sec. IX o decenni successivi) in Nelson, Manthe (nr. 44: I920). Si ricorderà come anche Collezione canonica in cinque libri uno dei principali testimoni dell'uso altomedievale della Lex Dei (supra, nt. 3I) - abbia la sua origine in Roma o nella zona di Roma.

circa la effettiva conoscenza della Lex Dei in età altomedievale. A dare loro forza e a confermare la circolazione di quel testo, sopravviene tuttavia un ulteriore argomento della cui solidità sarebbe invece difficile dubitare: la tradizione manoscritta. La Collatio è giunta sino a noi attraverso tre testimoni. Con tutta probabilità, anche se non direttamente, i tre codici discendono dal medesimo archetipo. ${ }^{36}$

Il più antico manoscritto della Collatio è conservato a Berlino (Berlin, DN, lat. fol. 269) ed è noto soprattutto perché contiene un fascicolo aggiunto con la fine delle Istituzioni e il principio del Digesto. Se questo singolo fascicolo pare databile alla prima metà del IX secolo, ${ }^{37}$ il resto del manoscritto, che contiene l'Epitome Iuliani, la Collatio e un'appendice di varî pezzi romanistici, dovrebbe essere precedente e risalire all'inizio di quel secolo o addirittura alla fine del precedente. L'origine è senz'altro italiana e si può pensare con una certa probabilità all'Italia settentrionale. ${ }^{38}$

Al secolo IX, ma più probabilmente alla fine, appartiene invece il secondo manoscritto della Lex Dei: il ms. Wien, ÖNB 2 I60. Contiene grosso modo i medesimi testi del berlinese e può dirsi anch'esso italiano. Indizî paleografici sono sembrati anzi in tempi recenti ricondurlo più precisamente alla zona di Roma. ${ }^{39}$

Il terzo e ultimo codice è quello conservato nella Biblioteca del Capitolo di Vercelli con la segnatura I22. Benché questo manoscritto sia stato copiato uno o due secoli dopo i precedenti (sec. XI), ad essi è tuttavia assai vicino per il contenuto. ${ }^{4 \circ} \mathrm{Di}$ nuovo ritroviamo assieme alla Collatio, l'Epitome di Giuliano e una serie di testi molto simile a quella già incontrata nei codici di Berlino e Vienna. Come il viennese, poi, anche il vercellese sembra esser stato copiato in area romana. ${ }^{4^{\mathrm{I}}}$ Sul finire del sec. XI appartenne a un privato, un tal Ambrosius iudex, con tutta probabilità un lombardo, che vi fece alcune annotazioni e vi aggiunse, in calce a uno stemma cognationum, il cap. I 53 dell'Editto di Rotari, anch'esso relativo ai gradi di cognazione. ${ }^{\mathbf{4}}$

Tre testimoni - è questo un dato che occorre sottolineare - non sono affatto pochi per quell'epoca. Soprattutto se si considera come molte opere giuridiche dell'alto Medioevo, assai più frequentate dai medievisti, si siano conservate in un unico esemplare. ${ }^{43}$ Poiché è da escludere che i manoscritti - e in particolare quelli giuridici - si copiassero per semplice diletto, un certo interesse per questo testo è quindi senz'altro da ammettere. ${ }^{44}$ Volendo tornare

42 Cfr. nuovamente Ciaralli (nr. 4) ntt. 50-54.

43 Si pensi, per fare solo alcuni esempî, alla Summa Perusina, alla Lex romana canonice compta, agli Excerpta Bobiensia, alla Glosse di Torino, Colonia e Pistoia, nonché alla lombarda Expositio ad Librum papiensem.

44 Non mi sembra sostenibile l'opinione espressa dal Volterra (nr. 63: 38 ) secondo cui i tre manoscritti (i quali, si ricordi, contenevano, oltre la Lex Dei, la usatissima Epitome Iuliani) sarebbero rimasti »sempre nascosti agli occhi dei giuristi «. Il contrario è invece dimostrabile almeno per il codice vercellese in cui il testo della Lex Dei si presenta corredato di glosse (oltre a quelle già trascritte dal Mommsen [nr. 42: I I I] che si trovano rispettivamente nei foll. I63r inf., I65r sup., I67r sup. et 
alla domanda da cui eravamo partiti, l'esame in particolare del codice vercellese non solamente ci rassicura sulla circolazione della Collatio nell'epoca che precede immediatamente Irnerio, ma pare oltretutto ricondurci in quel medesimo ambiente di giudici padani in cui il racconto di Radulfus Niger colloca appunto il magister Peppo.

3. Riconosciuti dunque l'effettiva circolazione e l'impiego della Lex Dei nei secoli altomedievali, è ora il momento di interrogarsi sui motivi che decretarono una simile fortuna per quell'operetta tardoantica.

Non è certo il caso di affrontare qui, per l'ennesima volta, una questione storiografica tanto complessa - e forse addirittura irrisolvibile - quale è quella sull'origine della Lex Dei. ${ }^{45}$ Tuttavia, proprio al fine di meglio comprendere quella singolare fortuna, può essere utile riportare alla memoria una vecchissima tesi del Bluhme secondo la quale la Collatio sarebbe stata concepita per essere inserita tra le fonti giuridiche impiegate dagli ecclesiastici nell'episcopalis audientia. ${ }^{46} \mathrm{Il}$ suo contenuto e ancor più la medesima struttura ben si accordano, in effetti, con una simile finalità pratica. Non sappiamo se, così come ci è pervenuta, l'opera sia da considerarsi ultimata. Essa si presenta comunque come una sòrta di manualetto di diritto penale. Il confronto fra le norme del Pentateuco e quelle romane si sviluppa seguendo il filo conduttore dei comandamenti penalistici del Decalogo $(\mathrm{V}-\mathrm{X}=$ Exod. 20.13-I7 e Deut. 5.I7-2I). ${ }^{47} \mathrm{La}$ corrispondenza è anzi ancor più evidente nella Lex Dei di quanto non sarà, più tardi, negli stessi Libri Poenitentiales altomedievali. ${ }^{4}$

La tesi del Bluhme, a dire il vero, ha trovato l'opposizione di autorevoli studiosi, i quali hanno piuttosto sostenuto, e continuano a sostenere, l'idea di una origine giudaica dell'opera. Come già altri hanno osservato, tuttavia, contro la paternità ebraica della Lex Dei depone gravemente l'assenza in essa di ogni riferimento alla Torah orale $^{49}$ e al Talmud..$^{\circ}$ Per gli ebrei del IV e V secolo, entrambi costituivano infatti consolidati e imprescindibili complementi nella interpretazione e applicazione della legge mosaica. ${ }^{5 \mathbf{I}}$

D'altro canto, i maggiori ostacoli alla ipotesi di una origine cristiana dell'opera risiederebbero nel fatto che l'autore della Collatio non solo omette di avvalersi della legislazione costantiniana $e$, in genere, di quella degli altri imperatori cristiani, ma

inf., I78va e I 80 vb, si può segnalare anche un intervento sul testo di Coll. 8.7.2 a fol. I74rb) e di appendici (foll. I $86 \mathrm{r}-\mathrm{v}$ ) assai interessanti e indicative del contesto e della finalità che fecero da sfondo alla stesura del codice (cfr. in partic. CONRAT; nr. 6: 3I 3-4). Decisiva, direi, è poi la circostanza che il medesimo codice vercellese sia stato posseduto da un pratico (il index Ambrosius).
45 Si può invece rinviare alle ampie disamine di Nelson (nr. 45: IO4I I6), LIEBS (nr. 34: I 62-I74), BARONe-Adesi (nr. I: 7-24) e SCHRAGE (nr. 55:329-336). 46 Bluhme (nr. 2) 3 IO.

47 I primi tre titoli sviluppano infatti il quinto comandamento (non occides); i titoli IV, V e VI accorpano sistematicamente i divieti posti dal sesto e dal nono comandamento (non moechaberis; non concupis- ces uxorem proximi tui); il tit. VII coincide con il settimo comandamento (non furtum facies); i titoli VIII e IX corrispondono al comandamento ottavo (non loqueris contra proximum tuum falsum testimonium); i titoli dal X al XV, finalmente, integrano, più di quanto non appaia prima facie, le fattispecie comprese nell'ultimo comandamento (non concupisces domum proximi tui non servum non ancillam non bovem non asinum nec omnia quae illius sunt). Il XVI titolo sembrerebbe invece iniziare la trattazione delle materie privatistiche. Può anche darsi che l'autore si sia interrotto a questo punto per via dell'impossibilità di proseguire la comparazione (così Nelson; nr. 45: I I $3-$ 4). Si può peraltro osservare come anche quell'ultimo titolo, in ogni caso, sarebbe stato di grande utilità ai vescovi nello svolgimento della loro funzione di giudici o arbitri. Sulla generale rispondenza della partizione della Lex Dei al Decalogo, v. comunque già CoNRAT (nr. 6: 88 nt. 2), MOMMSEN (nr. 42: I 29) e LieBs (nr. 34: I7I); contra invece LAURIA (nr. 29: 260) e Nelson (nr. 45: II4-6).

48 Cfr. Conrat (nr. 6) 88-89 nt. 2.

49 Anch'essa, secondo la tradizione ebraica, ricevuta da Mosè sul monte Sinai. Cfr. in proposito Cohen (nr. 5: I 85-6) e, più recentemente, LEANEY (nr. 30: partic. I 87 e sgg.).

50 Così soprattutto SmITs (nr. 59: I $82-3)$ e, più recentemente, anche Pieler (nr. 50: 495-6). Anche la circostanza della sistematica sostituzione del termine sinagoga tutte le volte che questo comparisse nei testi della Bibbia citati - circostanza già notata dal VolTERRA (nr. 63: 82-84) e da lui considerata quale ulteriore argomento a favore della paternità ebraica - mi pare in realtà più facilmente spiegabile pensando a un autore cristiano.

5 I Gli esempi riportati dal CoHEN (nr. 5: ı 86) chiariscono anzi come proprio nelle conversazioni con non ebrei i rabbini si fermassero a sottolineare l'importanza della Torah orale. 
52 Si tratta di Coll. 5.3 ove è appunto riportata una costituzione di Teodosio I del 390.

53 Cfr. p. es. Volterra (nr. 63) $97 \mathrm{e}$ sgg. Il tentativo più serio in questo senso mi sembra quello condotto da Masi (nr. 38). Sul punto, diffusamente, Liebs (nr. 34: I65 e sgg.).

54 Questa tesi - fatta propria tra gli altri dallo Hyamson (nr. 24) e ripresa più di recente dal LAURIA (nr. 29: 258) e dal LieBs (nr. 34: I70) - si fonda tuttavia principalmente su un unico passo (Coll. 7.I.I: "... scitote, iuris consulti, quia Moyses prius hoc statuit ... «). Tale passo potrebbe effettivamente essere come suggerisce il Pieler (nr. 50: 496-7) una glossa a Coll. 7.3.2 fatta successivamente scivolare nel testo. Occorre tuttavia tener presente che essa, in fondo, non fa che ribadire una convizione spesso ripetuta nelle opere patristiche (cfr. p. es. Tertullianus, Apolog., 45.4: "... ipsas leges quoque vestras ... de divina lege, ut antiquiore, formam mutuatas. Diximus iam de Moysi aetate «) e che potrebbe esser stata tranquillamente indirizzata anche a giureconsulti di fede cristiana, magari per blandire il loro orgoglio religioso che poteva anche smarrirsi proprio nel confronto tra la scarna normativa mosaica e le norme romane di livello tecnico indiscutibilmente superiore.

55 Cfr. Schrage (nr. 55:344-5) e Cortese (nr. 8: II, I3). Il Pieler (nr. 50: 498), da parte sua, aveva già ipotizzato che l'autore della

nemmeno pare considerare i principî evangelici. Non si tratta però di ostacoli insormontabili.

Riguardo alla prima omissione, va per prima cosa sottolineato come essa non sia assoluta. L'esistenza di almeno una eccezione ${ }^{52}$ comporterebbe infatti la necessità di credere a interpolazioni successive, sempre difficili da dimostrare. ${ }^{53}$ Soprattutto, però, un tale atteggiamento appare illogico, in un cristiano, solo ritenendo che il vero intento di questa operetta fosse quello di dimostrare ai pagani la maggior antichità e quindi autorità della legge mosaica rispetto al diritto romano. ${ }^{54}$ Nient'affatto strano apparirebbe invece qualora si ammettesse che scopo precipuo dell'autore della Collatio fosse piuttosto quello di rassicurare i membri delle comunità cristiane circa l'opportunità di ricorrere utilmente al diritto 'pagano di Roma senza per questo incorrere in peccato. ${ }^{55}$

Per quanto poi attiene alla circostanza che l'autore della Lex Dei sembri prendere in considerazione unicamente i principî veterotestamentarî, occorre ricordare come una antichissima tradizione - che può farsi risalire sino all'apostolo Paolo ${ }^{56}$ - individuasse la Legge nei precetti imposti da Dio agli uomini a causa delle loro trasgressioni e affinché si astenessero dal peccare. Quei precetti andavano appunto riconosciuti nel Decalogo e nel Pentateuco. Come il Vangelo di Matteo precisava e lo stesso Paolo aveva ribadito, Cristo non era venuto ad abolire tale Legge ma a completarla e rinforzarla. ${ }^{57}$ Il Suo insegnamento, in particolare, avrebbe dovuto consentire di distinguere la vera Legge (la lex simplex o prima lex che il Signore Iddio aveva dettato antequam populus vitulum faceret et ad idolatriam convertetur) da tutta quella serie di vincula secundationis (o secundatio legis) che Dio era stato costretto a imporre aggiuntivamente, in séguito al tradimento del Suo popolo. ${ }^{58}$ L'antica legge mosaica, pertanto, non poteva affatto dirsi superata. Quanto di essa il Figlio non aveva espressamente corretto ma confermato, manteneva quindi intatta la autorità del Padre e la immediata cogenza che appunto le derivava dal proprio carattere divino. ${ }^{59}$ Lex ed evangelium si profilavano pertanto, agli occhi dei cristiani, come elementi distinti, come i due termini di un'endiadi. E come tali essi furono ininterrottamente considerati sino ai tempi del Decretum grazianeo ${ }^{60}$ e oltre.

La Collatio non si pone al di fuori di questa lunghissima tradizione. Semplicemente essa si preoccupa solo del primo termine dal momento che solo i precetti dell'antico Testamento - e non

Collatio potesse avere avuto in mente di »integrare il diritto romano nel cristianesimo".

56 Ad Galat., 3.I9.

57 "Non veni destruere legem neque prophetas, sed adimplere (Math. 5.I7). Cfr. anche Paolo, Ad Rom., $3 \cdot 3$ I e I0.4.

58 Didascalia Apostolorum (nr. I8: 6.6.9: "Lex autem est, quae locutus est Dominus Deus, antequam populus vitulum faceret et ad ido- latriam convertetur, id est decalogus et iudicia «). Cfr. inoltre 6.I 5.2 e sgg.: "... Lex ergo est indestructibilis, secundatio autem legis temporalis ... « e ancóra 6.I6.2 e 6.I7-I 8 .

59 Si vedano in proposito anche gli esempî riprodotti dal LAURIA (nr. 29: 265 e sgg.; particolarmente significativa l'iconografia, ivi, 27I-273).

60 Decr., Dictum post I. 6.3 . 
quelli evangelici - hanno un autentico carattere normativo. Di nuovo, la comprensione diviene più facile se si accetta la tesi di una redazione della Lex Dei con una duplice finalità, al tempo stesso apologetica (a favore dell'antico diritto di Roma) e pratica (offrire un sussidio ai vescovi nella loro attività di giudici).

Le prime comunità giudaico-cristiane, come è noto, erano caratterizzate dalle figure carismatiche dei rispettivi vescovi. A questi si affidarono sin dall'inizio anche per risolvere tutti $\mathrm{i}$ problemi di carattere latamente giuridico. In breve, i vescovi furono chiamati non solo a promuovere e controllare la disciplina dei fedeli, ma anche a fungere da autorevoli arbitri tutte le volte che lo sscandalo di una controversia minacciasse di inquinare la vita della singola comunità. ${ }^{6 \mathbf{I}}$ Poiché l'impostazione paradossale e antigiuridica del Vangelo non consentiva loro di far fronte a questo tipo di problemi, i vescovi trovarono allora naturale rivolgersi al vecchio Testamento che proprio in quei secoli la rilettura patristica andava >depurando o, se si preferisce, scristianizzandor. Non a caso l'ignoto autore della Didascalia Apostolorum (attivo in Siria o in Palestina nella prima metà del III sec.) sottolinea che la Legge (ed egli intende con ciò Decalogo e Pentateuco) »vocata est specialiter propter iudicia ${ }^{\mathbf{6}}{ }^{\mathbf{2}}$

Anche quando, dopo gli editti di Galerio e Costantino, i cristiani poterono ormai sentirsi a pieno titolo cittadini dell'Impero, essi continuarono tuttavia a preferire l'audientia dei loro presuli a quella dei magistrati pubblici. Il rapido diffondersi del cristianesimo a tutti i ceti della popolazione rese considerevolmente più gravosa per i vescovi questa incombenza sia in termini quantitativi che qualitativi. La legge mosaica, privata della plurisecolare e raffinata interpretazione rabbinica, si presentava però piuttosto scarna e, nella sua rigidità, alquanto malagevole: in una parola, insufficiente. Quando ancóra le persecuzioni non erano cessate, l'autore della Didascalia Apostolorum sembra già essere consapevole di questa carenza. Nel tentativo di trovare una soluzione, egli giunge a elaborare una teoria che, per i suoi tempi, è senz'altro originale: Cristo è venuto appunto a completare e correggere la legge mosaica salvando quanto in esso vi era di buono e cancellando quanto invece era ormai divenuto inutile. Ma ciò ha fatto non solo attraverso il Suo diretto insegnamento, bensì indirettamente, ispirando cioè gli stessi legislatori romani. ${ }^{6}$ Addirittura, quasi a voler anticipare la Collatio, l'autore della Didascalia

6I Su ciò, recentemente, VISMARA (nr. 62: 3-34) e CONDORELLI (nr. 7: 45-83). Utile anche SCIPIONI (nr. 57).

62 Così in partic. Didasc. 6.I6.I. Il compito di leggere il vecchio Testamento alla luce del messaggio evangelico - e al contempo di istruire i vescovi circa la sua utilizzazione nella pratica >giudiziaria è proprio fra quelli che l'ignoto autore della Didascalia assume su di sé; cfr. SCIPIONI (nr. 57) 202 e sgg.

63 Val la pena di rileggere questo passo che mi sembra sinora trascurato dalla storiografia (Didasc. 6.I9.I-2): "Dominus vero noster et salvator veniens et similitudines implevit et parabolas ostendit, et ea, quae salvant, docuit, et ae, quae nihil iuvant, destruxit, et ea, quae non salvant, solvit, non solum per semet ipsum docens, set et per Romanos inspirans ... Nam et Romani lege utuntur, secundationem autem praetermiserunt, propterea et <eorum imperium> confirmatum est. Tu autem, si hodie sub secundationes desideras esse Romanis imperantibus, quae sunt secundationis facere non potes: neque enim lapidare malignos neque interficere idolatras neque ministeria sacrificiorum facere ... « 
chiarisce il proprio ragionamento facendo l'esempio dell'omicidio e richiamando quindi il quinto comandamento (Didasc. 6.19.5):

In Lege dicit: Non occides. Si quis ergo interfecerit, a lege per Romanos condemnatur et sub Lege est.

È facile credere che l'insegnamento della Didascalia abbia potuto incontrare qualche opposizione nell'ancor giovane mondo cristiano. In esso, si sa, non mancavano posizioni più intransigenti decise a rifiutare ogni deviazione dalle Scritture, ogni commistione, perfino ogni contatto, con ciò che sapesse anche lontanamente di pagano. La crisi delle persecuzioni dioclezianee, in particolare, doveva aver lasciato il segno sviluppando in molti una fortissima avversione nei confronti dello Stato romano e del suo diritto. Furono allora soprattutto personaggi come Ambrogio di Milano e Agostino di Ippona a raccogliere, tra IV e V sec., l'intuizione che un secolo prima aveva avuto lo sconosciuto scrittore orientale. ${ }^{64}$

Nulla impedisce di credere che un simile scenario e analoghe motivazioni possano aver fatto da sfondo anche al lavoro di chi, più o meno negli stessi decenni, si accingeva alla stesura della Collatio legum Mosaicarum et Romanarum.

4. Ma lasciamo finalmente l'epoca del Tardoantico e torniamo a interrogarci sui motivi che, trascorsi alcuni secoli, determinarono in pratica una seconda vita del nostro testo. Le condizioni storiche, il contesto sociale e culturale sono evidentemente mutati. Eppure, con i necessarî aggiustamenti e ricalibrature, entrambe le motivazioni che si è creduto di poter riconoscere dietro la compilazione della Collatio potrebbero aver conservato la loro sostanziale validità anche nei secoli successivi.

Si è detto che la redazione della Collatio potrebbe esser stata concepita in risposta al pressante bisogno di un testo che riuscisse utile agli ecclesiastici nel disbrigo della loro attività di giudici. Nell'età altomedievale, è cosa nota, le attribuzioni giudiziarie già assicurate ai vescovi del tardo mondo romano furono non solo mantenute ma spesso anche ampliate. ${ }^{65} \mathrm{Il}$ fenomeno è comune alla Spagna visigota, alla Francia merovingia e all'Italia longobarda.

$\mathrm{Ma}$ fu soprattutto in epoca carolingia che questo ruolo dei presuli assunse una importanza e una dimensione del tutto peculiari. Un capitolare di Carlo Magno dell'802/803 rivela, per esempio, l'impegno della corona nel tentativo di imporre la gene-

64 Per Ambrogio si può rinviare a questo proposito all'episodio del processo alla vergine Indicia da lui stesso didascalicamente riferito in due epistole del 380 (cfr., da ultimo, Loschiavo [nr. 37]). Relativamente al vescovo africano (v. anche infra nt. 75) può invece ricordarsi in particolare l'epistola 24 (su cui Lepelley; nr. 32). Cfr. inoltre VISMARA (nr. 62: 59-82 per Ambrogio e 97-I 28 per Agostino).

65 Ai lavori già precedentemente citati di Vismara (nr. 62: 197 e sgg.) e Condorelli (nr. 7: IO7 e sgg.), devono aggiungersi HARTMANN (nr. 22) e GAUdemet (nr. I9: I9O e sgg.). 
rale osservanza del Decalogo. ${ }^{66}$ Ed è esplicitamente ai vescovi che viene rimesso il compito di inquirere quei mala quae contraria sunt Deo, quae in sacris scripturis leguntur, quae christiani devitare debent. Fu, molto probabilmente, proprio in ottemperanza a questo ordine ideale che il vescovo di Chur, Remedio - un personaggio di cui è nota la vicinanza ad Alcuino - si decise a redigere una breve raccolta di Capitula a contenuto prevalentemente penalisitico e chiaramente ispirata al Decalogo se non, anch'essa, direttamente improntata alla Collatio. ${ }^{67}$ In un contesto del genere, non sarebbe certo sorprendente il veder raffiorare la vecchia $L e x D e i$ dalla polvere di qualche biblioteca. Non vi è dubbio, comunque, che una tale riapparizione avrebbe risposto assai bene al nuovo clima culturale innaugurato con la renovatio Imperii.

Le citazioni altomedievali della Collatio e la sua stessa tradizione manoscritta, se da un lato parrebbero confermare questa supposizione, ${ }^{68}$ dall'altro rivelano anche l'esistenza di un fortissimo legame tra la Lex Dei e l'Epitome di Giuliano. ${ }^{69}$ Più esattamente la Collatio risulta essere una componente stabile della cosiddetta appendice B che i manoscritti presentano spesso congiunta alla collezione di Novellae epitomate. ${ }^{70}$ L'una e l'altra, Collatio ed Epitome Iuliani, potrebbero allora esser state ricomprese in una delle versioni in cui dovette circolare quella enigmatica Lex Romana tante volte echeggiata nelle fonti. ${ }^{7 \mathbf{T}}$ Di quest'ultima, anzi, la Collatio unitamente al Dictatum de consiliariis ${ }^{72}$ - altra componente stabile dell'appendice all'Epitome di cui s'è detto potrebbe aver costituito le parte processual-penalistica.

Ma nemmeno l'altra motivazione che si è proposta - la necessaria funzione di supporto e di integrazione che il diritto romano avrebbe avuto nei confronti dei troppo scarni precetti divini - doveva aver perso ogni attualità. Naturalmente, i termini si ponevano in maniera differente. All'epoca di Ambrogio e Agostino si era trattato di difendere dai rigorismi gnostici e volgere a proficuo impiego la grande esperienza giuridica romana anteriore a Costantino. La renovatio Imperii imponeva ora la necessità di rinsaldare quello spirito di collaborazione tra i due poteri universali affievolitosi con la crisi dell'Impero occidentale e il progressivo distacco della chiesa di Roma da Costantinopoli. Chiesa e Impero, è appena il caso di ricordarlo, dovevano apparire come due facce di un'unica medaglia. Di tale ideologia, a lungo portante nei secoli dell'alto Medioevo, il concetto dell'utraque lex, dell'una e dell'altra

66 Ed. MGH, Capitularia, I I70, 33 e sgg. (c. I): »Ut episcopi circumeant parrochias sibi commissas et ibi inquirendi studium habeant de incestu, de patricidiis, fratricidiis, adulteriis, cenodoxiis et alia mala quae contraria sunt Deo, quae in sacris scripturis leguntur quae christiani devitare debent «; testo citato da Hartmann (nr. 22: I09).
67 In proposito, cfr. Soliva (nr. 60). Si può osservare come il breve testo - che Remedio (c. I2 in fi.) raccomanda esplicitamente al suo clero di portare sempre con sé e di leggere pubblicamente almeno due volte al mese - ci sia stato trasmesso da un manoscritto degli inizî del IX sec. il quale contiene, oltre alla Lex Romana Curiensis di cui costituisce una sòrta di appendice, anche l'Epitome Iuliani (v. infra). Oltre che all'edizione per i Monumenta (nr. 65) si può fare riferimento a quella successivamente approntata da MEYERMarthaler (nr. 39: 645 e sgg.). Alla studiosa svizzera (nr. 40) si deve anche il contributo sinora più approfondito su questo testo (per la corrispondenza alla legislazione carolingia dell'802, ivi 92).

68 Come si è visto, sia i più antichi testimoni manoscritti sia le più antiche citazioni rimandano infatti all'età carolingia o immediatamente post-carolingia.

69 Si può osservare come in due manoscritti su tre, l'elenco dei capitoli in essi contenuti prosegue senza distinzione trascorrendo da quelli dell'Epitome a quelli della Lex Dei.

70 Conrat (nr. 6: 87-8 e I 29-30); Nelson, Manthe (nr. 44: 9 e I 4); SIEMS (nr. 58: I77-8).

7 I In proprosito si veda ora soprattutto CoRTese (nr. 8: I, 245-247).

72 Si tratta di un piccolo repertorio di passi giustinianei indubbiamente rivolto alla prassi forense e a quella del foro episcopale in particolare. È stato edito da HäNEL (nr. 2I: xlix e sg. e I98-20I). Cfr. inoltre CONRAT (nr. 6: I37-I4O). 
$73 \mathrm{Su}$ di esso v. soprattutto CORTESE (nr. Io e nr. 8: I, 387 ; II 57-8 e 80-I).

74 Attaccatissima all'Epitome Iulia$n i$, tanto non solo da preservarne il testo dall'oblío ma da promuoverne anche la diffusione oltralpe, fu, com'è noto la Chiesa (cfr. Cortese; nr. 8: I, 242 e sgg. e partic. 24394 e 245 IO2). Un simile interesse aveva ragioni in pari tempo pratiche e ideologiche. Certamente le Novellae giustinianee, disciplinando numerosi aspetti >secolari< della vita della Chiesa stessa, si presentavano come un prezioso strumento normativo in sé. Le molte disposizioni novellari sull'ordinamento dei chierici, sui monaci, su nosocomi e orfanotrofi, sul matrimonio potevano contemporaneamente servire come ottimi esempî da additare ai sovrani carolingi e poi sassoni per stimolarne l'emulazione. Può essere utile, a questo proposito, ricordare come ricorra spesso nella tradizione altomedievale dell'Epitome - o meglio delle serie di excerpta di capitoli dell'Epito$m e$ - l'accenno a una pretesa collaborazione del clero alla attività normativa di Giustiniano: cfr. PATETTA (nr. 48: I 2). Un significativo riscontro nei capitularia carolingi si può ritrovare nella rubrica del c. 96 (aa. 782-8 IO; ed. A. Boretius - V. Krause, in MGH, Legum sectio II. Capitularia regum Francorum, I, Hannoverae 1883 ): »Capitula cum Italiae episcopis deliberata «.

75 Incmaro propugna ripetutamente nei suoi scritti l'opportunità per la Chiesa di tener conto della normativa laica. Addirittura ripropone una opinione di Agostino circa la perdurante validità di quelle leggi romane che, pur anteriori alla venuta di Cristo, possono nondimeno dirsi ispirate da Dio (PL I25, IOI 5): »De legibus a quibusdam imperatoribus male constitutis, a quibusdam vero bene constitutis, sanctus Augustinus ad Bonifacium dicit: Imperatores quando pro falsitate contra veritatem constituunt malas leges, probantur bene credentes et coronantur perseverantes. Quando

legge, costituiva una componente fondamentale. ${ }^{73} \mathrm{Al}$ pari dell'Epitome Iuliani, ${ }^{74}$ la Collatio poteva anch'essa rappresentare una espressione mirabile e concreta di quella visione.

Ben presto, tuttavia, almeno una certa parte del clero avrebbe cominciato a non riconoscersi più in quell'ideale. Si trattava in particolare di quella parte presso cui più facilmente attecchivano le istanze riformistiche a favore di una sempre maggiore autonomia 'politicar della Chiesa. Può non esser stato semplicemente un caso, allora, se tutti coloro che, a partire dall'età carolingia, rivelano la conoscenza e l'uso della Lex Dei ci appaiono schierati sul fronte maggiormente ostile alla riformar.

Attestato su quella linea, per cominciare, era senz'altro il potente arcivescovo di Reims. Di Incmaro sappiamo infatti che, mentre da un lato si ergeva a grande oppositore delle falsificazioni pseudoisidoriane, dall'altro si professava convinto assertore della necessaria collaborazione tra i due poteri e della opportunità, per gli ecclesiastici, di conoscere e considerare anche la legge civile. ${ }^{75}$

In séguito, nei primi decenni dell'XI secolo, si trovarono sulle medesime posizioni anche $\mathrm{i}$ non pochi ecclesiastici italiani che decisero di sposare la causa imperiale e appoggiare il breve ma intenso sodalizio collaborativo instaurato tra Enrico II e Benedetto VIII. ${ }^{76}$ Tra di loro era certamente anche l'autore della Collectio canonum V librorum. ${ }^{77}$

Ancor più tardi, all'epoca cioè in cui Enrico IV eleggeva l'antipapa Clemente III e ispirava la stesura di polemici libelli intitolati De unitate ecclesiae conservanda ${ }^{78}$ è assai probabile che fosse schierato su quel versante anche il misterioso Pepo, bàiulus del Codice e delle Istituzioni giustinianee e nondimeno deciso propugnatore del sistema dell'utraque lex e, su questa base, anche abile persuasore del medesimo Enrico IV. ${ }^{79}$

Ma l'episodio del placito lombardo va evidentemente considerato alla stregua di un colpo di coda. Ben presto le idee riformistiche avrebbero preso decisamente il sopravvento. Di lì a poco si sarebbe affacciata sulla scena la rivoluzionaria figura di Irnerio. Per Pepo, per il suo vecchio mondo, persino per la memoria dei suoi insegnamenti, non ci sarebbe stato più alcuno spazio. Per la Collatio legum Mosaicarum et Romanarum era invece il momento di far ritorno nelle biblioteche e ricoprirsi nuovamente di polvere.

\section{Luca Loschiavo}

rentur saevientes et corriguntur intellegentes «; cfr. DEvisse (nr. II: I, 422 e sgg. e 549 e sgg., partic. 552463 ) e inoltre LoRTZ (nr. 35: I, 350-I) e GAUDEMET (nr. I9: I73-4).

76 Capitani (nr. 3: 237-25I).

77 Cfr. supra, nt. 3 I (e partic. FourNIER; nr. I7: 2773).

78 Capitani (nr. 3: 334 e sgg.).

79 Che Pepo fosse un ecclesiastico sembra probabile al CORTESE (nr. 8: II, 43-45) il quale nemmeno respinge del tutto la sardita identificazione col vescovo scismatico bolognese Pietro ( I085I096) suggerita alcuni anni fa dal Fiorelli (nr. I 5 ) sulla base di un racconto dell'umanista Sigismondo Ticci. Qui, in aggiunta, si può segnalare l'impressionante corrispondenza tra i contenuti dell'intervento peponiano al placito lombardo e un passo famoso 
Elenco della letteratura citata

I. BARONe-Adesi, G., L'età della "Lex Dei«, Napoli 1992

2. Blunme, F., Praefatio all'ediz. della Lex Dei da lui curata per il »Corpus Iur. Rom. Anteiust. ", I, Bonnae I84I, 3 IO-3 I I

3. Capitani, O., Storia dell'Italia medievale: 4IO-I2 I6, Bari-Roma I986

4. Ciaralli, A., Produzione manoscritta e trasmissione dei testi di natura giuridica fra XI e XII secolo. Due esempi, in: Juristische Buchproduktion im Mittelalter, V. Colli cur. (Studien zur europäischen Rechtsgeschichte, I 55 ) Frankfurt a. M. 2002, 7 I-IO3

5. Cohen, A., Il Talmud, Roma I935 (rist. Bari-Roma I999)

6. Conrat (Cohn), M., Geschichte der Quellen und Literatur des römischen Rechts im frühen Mittelalter, Leipzig I 89I (rist. Aalen I963)

7. Condorelli, O., Ordinare - Iudicare. Ricerche sulle potestà dei vescovi nella Chiesa antica e altomedievale (secoli II-IX), Roma I997

8. Cortese, E., Il diritto nella storia medievale, I-II, Roma 1995

9. Cortese, E., Tra glossa, commento e umanesimo, in: ID., "Scritti « (I. Birocchi - U. Petronio curr.), Spoleto I999, II, I049I094 (già in: "Studi Senesi «, 4I [1992] 458-503)

io. Cortese, E., Lex, aequitas, utrumque ius nella prima civilistica, in ID., »Scritti« (I. Birocchi U. Petronio curr.), Spoleto I999, II, IOI9-IO43 (già in: >Lex et iustitia nell'utrumque ius: radici antiche e prospettive attuali, Atti del VII coll. intern. romanistico-

del De ordine palatii scritto quasi duecento anni prima da Incmaro (ed. in MGH, Fontes iur. Germ. ant., 3, Hannoverae 1980 , cap. 2I): »Comites autem palatii inter caetera paene innumerabilia in hoc maxime sollicitudo erat, ut omnes contentiones legales, quae alibi ortae propter aequitatis iudicium palatium aggrediebantur, iuste ac rationabiliter determinaret seu perverse indicata ad aequitatis canonistico, I2.-I4.5.I988, Città del Vaticano I989, 95-I I9)

i I. Devisse, J., Hincmar, Archevêque de Reims: 845-882, I-III, Geneve I 975

I2. Dolcini, C., Postilla su Pepo e Irnerio, in: appendice alla ristampa di G. de Vergottini, Lo Studio di Bologna, l'Impero, il Papato, Spoleto I996, 83-100

I3. Dolezalek, G., Verzeichnis der Handschriften zum römischen Recht bis I600, I-IV, Frankfurt a.M. I 972

I4. Ferrary, J. L., Lex Cornelia de sicariis et veneficis, in: Athenaeum 79 (I99I) 4I7-434

I 5. Fiorelli, P., Clarum Bononiensium Lumen, in: Per Francesco Calasso. Studi degli allievi, Roma I978, 4I 5-459

i6. Fournier, P., Un groupe de recueils canoniques italiens des Xe et XIe siècles, (I9I6) ora in: ID., Mélanges de droit canonique, Aalen I983, II, 2I3-33 I

i7. Fournier, P. - Le Bras, G., Histoire des collections canoniques en occident, I, Paris I93 I (rist. Aalen I972)

I8. Funk, F. X., Didascalia et constitutiones apostolorum, I, Paderborn I905 (rist. Torino 1979)

I9. Gaudemet, J., Église et Cité. Histoire du droit canonique, Paris I994 (trad. ita., Storia del diritto canonico. Ecclesia et Civitas, Cinisello Balsamo I998)

20. HÄNEL, G., Lex Romana Visigothorum, Lipsiae I 848 (rist. Aalen I962)

2I. HäNEL, G., Iuliani epitome latina Novellarum Iustiniani, Leipzig I873 (rist. Osnabrück 1965)
22. Hartmann, W., Der Bischof als Richter. Zum geistlichen Gericht über kriminelle Vergehen von Laien im früheren Mittelalter (6.-II. Jahrhundert), in: Römische historische Mitteilungen 28 (I986) I03-I24 (trad. ita. in: Rivista di storia della Chiesa in Italia 40.2 [I986] 320-34I)

23. Herdlitczka, A., v. Talio, in: Pauli-Wissowa R.E. IV A.2 (I932) 2069-77

24. Hyamson, M., Mosaicarum et Romananrum legum Collatio with introduction, facsimile and transcription of the Berlin Code, translation notes and appaendices, Oxford I9I3

25. Kantorowicz, H.-Smalley, B., An English Theologian's View of Roman Law: Pepo, Irnerius, Ralph Niger, (1943), ora in: KaNTOROwICZ, H., Rechtshistorische Schriften, (a cura di H. Coing G. Immel) Karlsruhe 1970, 23 I244

26. KérY, L., Canonical Collections of the Early Middle Ages (ca. 400I I 40), Washington D.C. 1999

27. KoAL, V., Zur Überlieferungsgeschichte der Fünf-Bücher-Sammlung, in: Quellen, Kritik, Interpretation. Festgabe H. Mordek (a cura di T. M. Buck e aa.), Frankfurt a. M. e aa. 1999

28. KUnKeL, W., Untersuchungen zur Entwicklung des römischen Kriminalverfahrens in vorsullanischer Zeit, [Bayer. Akad. der Wiss., Phil.-hist. Kl. Ab., n. F., 56] München 1962

29. Lauria, M., Lex Dei, in: Studia et Documenta 5I (I985) 257-275 tramitem reduceret, ut et coram Deo propter iustitiam et coram hominibus propter legum observationem cunctis placeret. Si quid vero tale esset, quod leges mundanae hoc in suis diffinitionibus statutum non haberent aut secundum gentilium consuetudinem crudelius sancitum esset, quam christianitatis rectitudo vel sancta auctoritas merito non consentiret, hoc ad regis moderationem per- duceretur, ut ipse cum his, qui utramque legem nossent et Dei magis quam humanarum legum statuta metuerent, ita decerneret, ita stetueret, ut, ubi utrumque servari posset, utrumque servaretur, sin autem, lex saeculi merito comprimeretur, iustitia Dei conservaretur.« 
30. Leaney, A.R. C., The Jewish and Christian World. $200 \mathrm{BC}$ to AD 200, (Cambridge Commentaries on Writings of the Jewish and Christian World $200 \mathrm{BC}$ to $\mathrm{AD}$ 200, 7) Cambridge I984

3 I. Legendre, P., La Summa Institutionum >Iustiniani est in hoc opere , Ius Commune Sonderhefte, 2 Frankfurt a. M. 1973

32. LePelley, C., Liberté, colonat, esclavage d'après la lettre 24: la jurisdiction épiscopale sde liberali causa<, in: Les lettres de Saint Augustin découvertes par J. Divjak, Paris $1983,329-342$

33. Levy, E., Die römische Kapitalstrafe (I93I) ora in: ID., Gesammelte Schriften, II, Köln, Graz I963, 325 e sgg.

34. Liebs, D., Die Jurisprudenz im spätantiken Italien (260-640 n. Chr.), Berlin 1987

35. Lortz, J., Storia della Chiesa, trad. ita., I-II, Cinisello Balsamo I 992

36. Loschiavo L., "Secundum Peponem dicitur ... G. vero dicit «. In margine ad una nota etimologica da Pepo ad Ugolino, in: Riv. int. di diritto comune 6 (I995) 233 e sgg.

37. Loschiavo, L., Tra legge mosaica e diritto romano, in: A Ennio Cortese, Roma 200I, II, 269-284

38. Masi, A., Contributi alla datazione della "Collatio legum Mosaicarum et Romanarum «, in: Bullett. dell'Ist. di Dir. Rom., 64 (I96I) 285-32I

39. Meyer-Marthaler, E., Die Rechtsquellen des Kantons Graubünden, I, Lex Romana Curiensis, Aarau I959

40. Meyer-Marthaler, E., Die Gesetze des Bischofs Remedius von Chur, in: Zeitschr. f. Schweiz. Kirchengeschichte 44 (I950), 8 I-I IO e I6I-I 88

4I. Mommsen, T., Römisches Strafrecht, Leipzig I 899 (= Aalen I990)

42. Mommsen, T., Praefatio all'ed. della Collatio nella "Collectio librorum iuris anteiustiniani «, III, Berolini I 890

43. Mor C. G., La Bibbia e il diritto canonico, in: La Bibbia nell'alto
Medioevo (Settimane di studio del CISAM, X) Spoleto I963, I63I 79

44. Nelson, H. L. W. - Manthe, U., Gai Institutiones III I-87, (Freiburger Rechtsgesch. Abhandl., n. F. I 5 = Studia Gaiana VII) Berlin I 992

45. Nelson, H. L. W., Überlieferung, Aufbau und Stil von Gai Institutiones, Leiden I98 I

46. NicolaJ, G., Ambiti di copia e copisti giuridici in Italia (secc. V$\mathrm{XI}$ in.), in: A Ennio Cortese, Roma 200I, II, 478-496

47. Patetta, F., Sui frammenti di diritto germanico della Collezione Gaudenziana e della Lectio legum, ora in: ID., Studi sulle fonti giuridiche medievali, Torino I967, 86I-898 (già in: Archivo giuridico 53 [1 893] 3-40)

48. Patetta, F., Note sopra alcuni mss. delle Istituzioni di Gustiniano, con appendice di glosse inedite, in ID., Studi sulle fonti giuridiche medievali, Torino I967, 4 I-I 20 (già in: Bullettino dell'Ist. di Dir. Rom. 4 [I89I] I7-96)

49. Patetta, F., Contributi alla storia del diritto romano, in: ID., Studi sulle fonti giuridiche medievali, I 2 I-I 58 (già in: Bullettino dell'Ist. di Dir. Rom. 4 [I 89I] 249-286)

50. Pieler P. E., Lex Christiana, in: Akten des 26. Deutschen Rechtshistorikertages (Frankfurt a. M. 22.-26.9.1986) [Ius Commune Sonderhefte, 30 ] Frankfurt a.M. I $987,485-503$

5I. Rilinger, R., Humiliores - Honestiores. $\mathrm{Zu}$ einer sozialen $\mathrm{Di}$ chotomie im Strafrecht der römischen Kaiserzeit, München I 988

52. RöHLE R., Das Berliner Institutionen- und Digestenfragment ms. Lat. fol. n. 269, in: Bullettino dell'Ist. di Dir. Rom. 7I (I968) I 29-I73

53. Santalucia, B., Diritto e processo penale nell'antica Roma, 2a ed. Milano 1998

54. Schmugge L., "Codicis Iustiniani et Institutionum baiulus«. Eine neue Quelle zu Magister Pepo von Bologna, in: Ius Commune 6 (I977) I-9

55. Schrage, E., La date de la Collatio Legum Mosaicarum et Romanarum étudiée d'après les citations bibliques, in ID., "Non quia romanum sed quia ius", (Bibliotheca eruditorum, I7), Goldbach 1996, 329-345 (già in: Mélanges F. Wubbe, Fribourg I993, 40I-4I7)

56. Schultz, F., The Manuscripts of the Collatio legum mosaicarum et romanarum, in: Symbolae van Oven, Leyden I946, 313-333

57. Scipioni, L. I., Vescovo e popolo. L'esercizio dell'autorità nella chiesa primitiva (III secolo), [Vita e pensiero. Pubbl. dell'Univ. Cattolica del S. C. - Scienze religiose, 3 ] Milano I977

58. Siems, H., Handel und Wucher im Spiegel frühmittelalterlicher Rechtsquellen, MGH, Schriften Vol. 35, Hannover 1992

59. Smits, N., Mosaicarum et Romanarum legum collatio, Haarlem I934

6o. Soliva, C., Zu den Capitula des Bischofs Remedius von Chur aus dem beginnenden 9. Jahrhundert, in: »Nit anders denn liebs und guets « a cura di C. Sснотт et C. Soliva, Sigmaringen I986, I 67-I 72

6i. Supino-Martini, P., Roma e l'area grafica romanesca (secoli X-XII), Alessandria 1987

62. VIsmara, G., La giurisdizione civile dei vescovi, Milano 1995

63. Volterra, E., Collatio legum Mosaicarum et Romanarum, (Memoria della Reale Accademia dei Lincei; a. CCCXXVII [1930] ser. VI, vol. III, fasc. I) Roma I930

64. Wasserschleben, F. G. A. ed., Reginonis Prumiensis. Libri duo de synodalibus causis et disciplinis ecclesiasticis, Lipsiae I 840 [= Graz I964]

65. Zeumer, K. ed., Additamenta Codicis S. Galli, quae vulgo Capitula Remedii dicuntur, (MGH, Leges, V, 44I e sgg.) 